\title{
ION AND MOLECULE SENSORS USING MOLECULAR RECOGNITION IN LUMINESCENT, CONDUCTIVE POLYMERS
}

\author{
FY1997 YEAR-END PROGRESS REPORT \\ Michael R. Wasielewski \\ Chemistry Division \\ Argonne National Laboratory \\ Argonne, IL 60439
}

\section{Purpose}

The purpose of this project is to use molecular recognition strategies to develop sensor technology based on luminescent, conductive polymers that contain sites for binding specific molecules or ions in the presence of related molecules or ions. Selective binding of a particular molecule or ion of interest to these polymers will result in a large change in their luminescence and/or conductivity, which can be used to both qualitatively and quantitatively sense the presence of the bound molecules or ions. The main thrusts and accomplishments in the first year of this project involve developing polymer syntheses that yield conjugated polymers to which a wide variety of ligands for metal ion binding can be readily incorporated.

\section{Target Problem}

Research problems that will be addressed by this project include: 1) designing molecular recognition sites that are highly selective for the ions and/or molecules of interest in the presence of a large background of other chemical species, 2) finding ways to incorporate many different selective groups into a single polymer, 3) fabricating polymer films, strips, sheets, and coatings that can be applied to other materials, such as fiber optics and surfaces, 4) developing interfaces between the polymers and substrates that can be used to produce prototype arrays of many sensor elements for rapid multi-contaminant detection and quantitation, and 5) developing multiplexed data collection techniques to rapidly process the data obtained from many polymer sensors into a chemical profile of a waste stream or waste site in real time. 


\section{Benefits}

Molecule-selective sensors will prove very valuable for quantitation of hazardous materials in waste streams and in efforts to gauge the effectiveness of environmental remediation strategies at specific waste sites. It is important that sensor materials be cheap, easily manufactured, resistant to harsh chemical environments, sensitive to and selective for a variety of chemical species, physically small, and capable of modification as new needs arise. The technology that will be derived from the combination of luminescent, conductive polymers with sites designed for specific molecular recognition will fulfill these requirements. For example, the Weldon Springs, MO site will serve as a model site for tailoring specific sensors for determining ground water contamination. This site has a wide range of heavy metal, actinide, and organic contaminants that can be assessed by the development of new sensor technology based on molecular recognition. Argonne National Laboratory is the compliance contractor for the Weldon Springs site and is responsible for providing the primary support for evaluating environmental conditions to meet CIRCLA requirements. This role provides important insights, experiences, and personal contacts for sensor development for ground water applications.

\section{Technical Progress in FY1997}

Research into highly sensitive and selective molecular sensory materials has received considerable attention in recent years.' In particular, numerous efforts have been devoted to the design and construction of chemosensory systems that are capable of detecting metal ions in both a real-time and reversible fashion. '-' Among the systems reported to date, the vast majority constitute either crown ethers or their analogs,'

conjugated polymer-based ${ }^{6}$ super-molecules, or, more attractively. the combination of these two. ${ }^{7-12}$ The appeal of the latter type of system is that it makes use of not only the well-known selective binding of alkali or alkaline earth metal ions to crown ethers, but also the high sensitivity of conjugated polymers to external structural and/or electronic perturbations. The sensitivity of conjugated polymers to these perturbations results from association with the metal ions. 
There are few examples, on the other hand, that show the feasibility of making other metal ion sensitive and conjugation enhancement based, polymers. ${ }^{8,10,11}$ Heavy metals, such as transition metals, lanthanides, and importantly, actinides, provide the bulk of environmental metal contaminants. In addition, there are a large number of organic molecules that provide their own special hazards in the environment. It should be possible to develop a series of molecular recognition sites that specifically bind these contaminants to a conjugated polymer in a manner that provides sufficient perturbation of the structural and electronic properties of the polymer in order to use luminescence and/or conductivity changes within the polymer to detect the presence of the contaminants. Moreover, given the polymeric nature of the resultant sensor material, these polymers can be readily applied as coatings to substrates, such as fiber optics, to produce working sensors.

Fluorescent sensors can recognize specific cations by incorporating selective chelating agents. Raymond et al. have used the highly ferric-ion-specific microbial chelators, siderophores, ${ }^{13}$ as models for the synthesis of iron chelators for medical applications. ${ }^{14}$ An extension of this chemistry has led to the development of plutonium(IV)-specific complexing agents, which animal testing has shown to be practical for decorporation therapy." In each of these kinds of chemistry the ligand design can be combined with luminescent, conjugated polymers to produce novel sensor materials for these important metal contaminants.

Polymers that contain specific ligands and receptors for metals and organic molecules can be used in conjunction with fiber optic technology to detect trace amounts of environmental contaminants remotely. The use of fiber optics for chemical sensors is a new field that has attracted considerable interest over the past several years. The Walt group at Tufts University has developed a number of novel approaches that use fiber optics for chemical sensing? The combination of this technology with the new molecular recognition polymers proposed here will result in enhanced sensors for remote chemical detection of contaminants.

Single strands of transparent materials such as glass, plastic, or fused silica can propagate light over long distances with minimal attenuation. Since light undergoes 
thousands of reflections per meter as it is guided down the length of the fiber, the position at which an individual ray exits the fiber will not correspond to the position at which it entered the fiber. As a result, a single optical fiber cannot be used to transmit an image.

Individual optical fibers can be fused into a fiber bundle. Both rigid and flexible fiber-optic bundles are available in many lengths, diameters, geometries, and multifurcated combinations. Most light-delivery bundles are used only to transmit energy and contain unaligned optical fibers. In contrast, imaging fibers are fiber-optic bundles in which the relative spatial coordinates of the individual optical fibers are retained in a fixed arrangement (i.e., the position of each optical fiber is the same at the input and output ends). In these bundles, each optical fiber acts like a pixel with the pixel size determining the resolution of the transmitted image. A modem high resolution imaging fiber is comprised of between 3,000 and 100,000 individually cladded optical fibers in which the-diameter of each fiber within the bundle is on the order of 3-10 $\mu \mathrm{m}$.

We have prepared several polymers based on the incorporation of 2,2'-bipyridine into poly(phenylenevinylene) polymers. These polymers are designed to show significant changes in their optical properties upon chelation of metals as depicted in Figure 1. This scheme illustrates the concept behind the use of these polymers as indicators of the
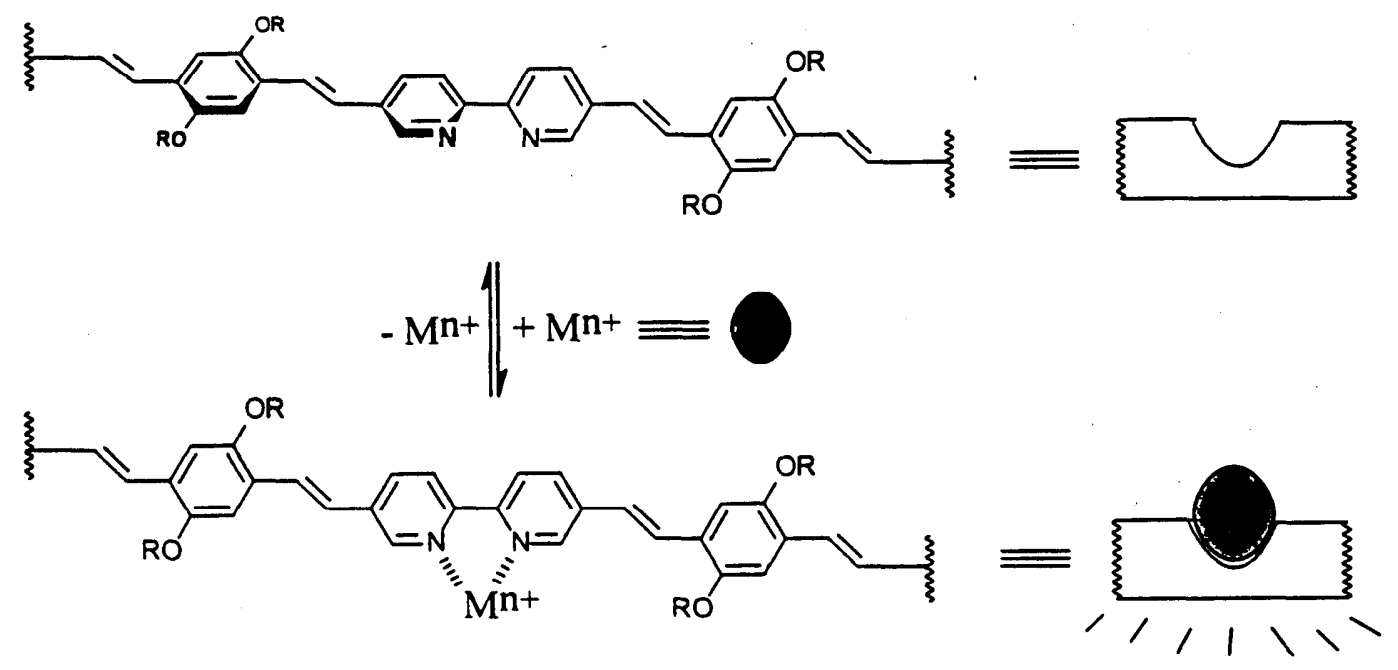

Figure 1. Metal binding results in conformational changes in the conjugated polymers. 
presence of the metal.

The central idea, illustrated in Figure 1, is to prepare a pseudoconjugated, ligandcontaining polymer that upon incorporating metal ions would undergo conformation adjustment, thus converting the initial partially conjugated polymer to a fully or near fully conjugated one. Such conjugation enhancement, along with the simultaneous electron density change caused by incorporating metal ions onto the backbone of polymers, dramatically changes the optical and conductivity properties of the polymer. These property changes are used to signal the presence of the metal ion analyte. Two specific

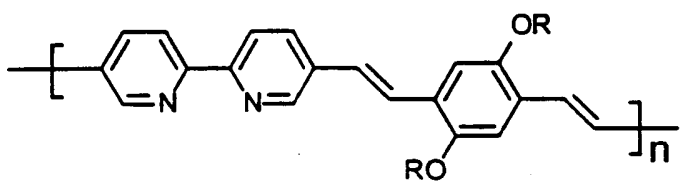

1

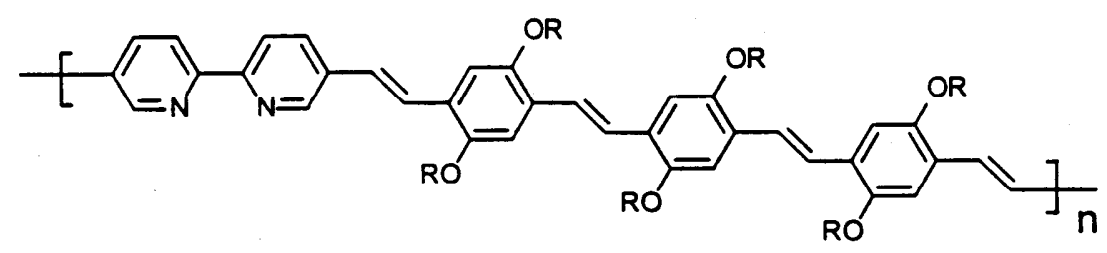

2

Figure 2. Structures of conjugated polymers 1 and $2, \mathrm{R}=2$ ethylhexyl.

polymers in this series are shown in polymer structures $\mathbf{1}$ and 2 in Figure 2. Here, the oligo-phenylenevinylenes are chosen as conjugated segments that are connected covalently to 2,2'-bipyridines at their 5,5'-positions. Since the phenylenevinylene block is designed also to be responsible for signal transduction, the different length, as in $\mathbf{1}$ and 2 , respectively, results in different responses.

The reason 2,2'-bipyridine was selected as a building block for these polymers is based on following considerations. It is well-known that 2,2'-bipyridine and its derivatives possess superb ability to coordinate a large number of metal ions. As such, the scope of metal ion sensors could be extended from currently well documented, crown ether based, alkali or alkaline earth metal ion sensitive systems to other metal ion sensitive ensembles. More intriguingly, given the fact that there is approximately $20^{\circ}$ a 
dihedral angle between two pyridine planes in a 2,2'-bipyridine when it is in its transoidlike conformation, ${ }^{14}$ such polymers are therefore not totally conjugated. When chelated with a metal ion, however, the coordination between the metal ion and bipyridine would
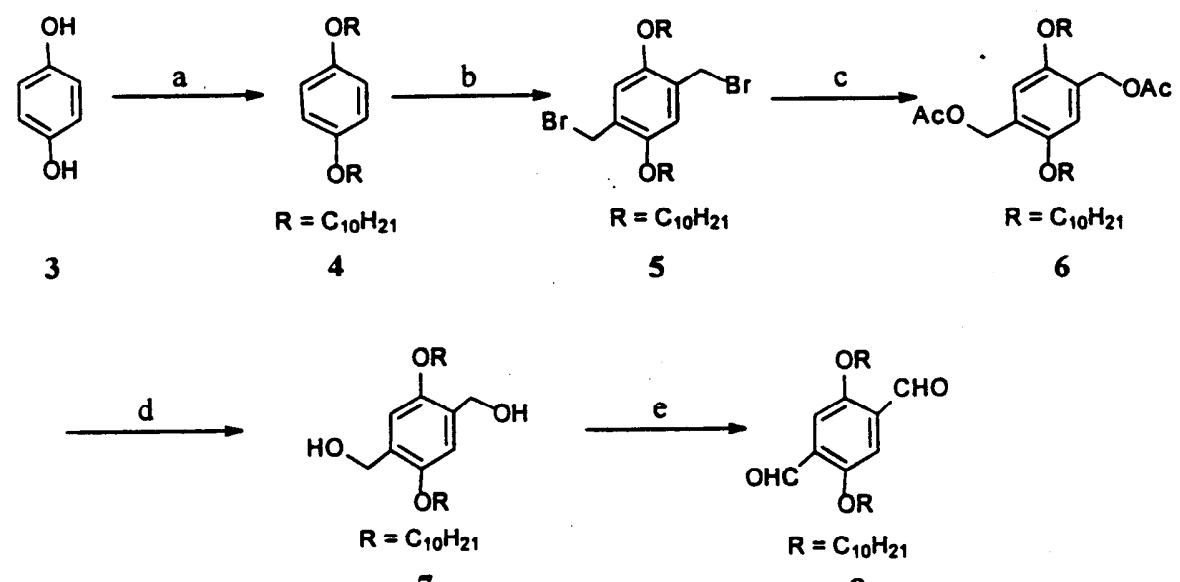

7

8

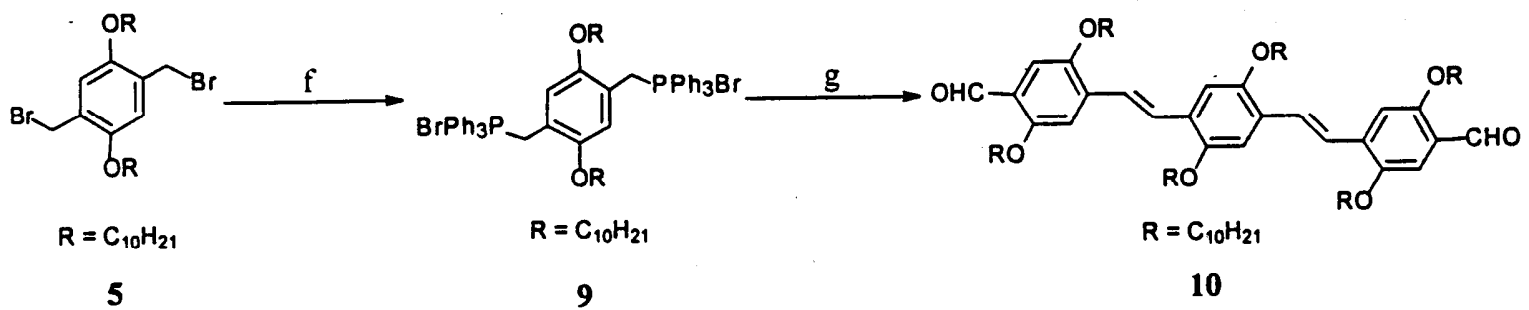

Figure 3. Synthetic scheme for polymer intermediates using Wittig coupling chemistry.

force the twisted conformation into a planar one, making the polymers fully conjugated. In addition, incorporating 2,2'-bipyridine as a recognition unit directly into the backbone, as an integral part of a conjugated polymer, is expected to give rise to a sensitive response upon metal ion binding.

Our initial strategies employed for synthesizing polymers 1 and 2 are based upon Wittig-type ethylenic group formation methodology. To this end, the corresponding diphosphonium salt and dialdehyde precursors were prepared first. Figure 3 illustrates the synthetic sequences for making dialdehydes 8 and 10. Specifically, heating 1,4hydroquinone 3 with 1-bromodecane in acetonitrile at reflux in the presence of $\mathrm{K}_{2} \mathrm{CO}_{3}$ readily afforded 1,4-bis-decyloxybenzene 4 in $85 \%$ yield. Subsequent bromomethylation of 4 , using a general bromomethylation procedure for aromatic compounds," then 


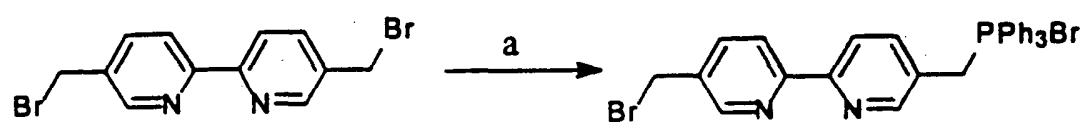

11

12<smiles>[R20]c1cc(C=Cc2cc([R20])c(C([Y])([R])C=O)cc2[2H])c(O)cc1C=O</smiles>

12

$x=0: 8$

$x=2: 10$

$\mathrm{R}=\mathrm{C}_{10} \mathrm{H}_{21}$
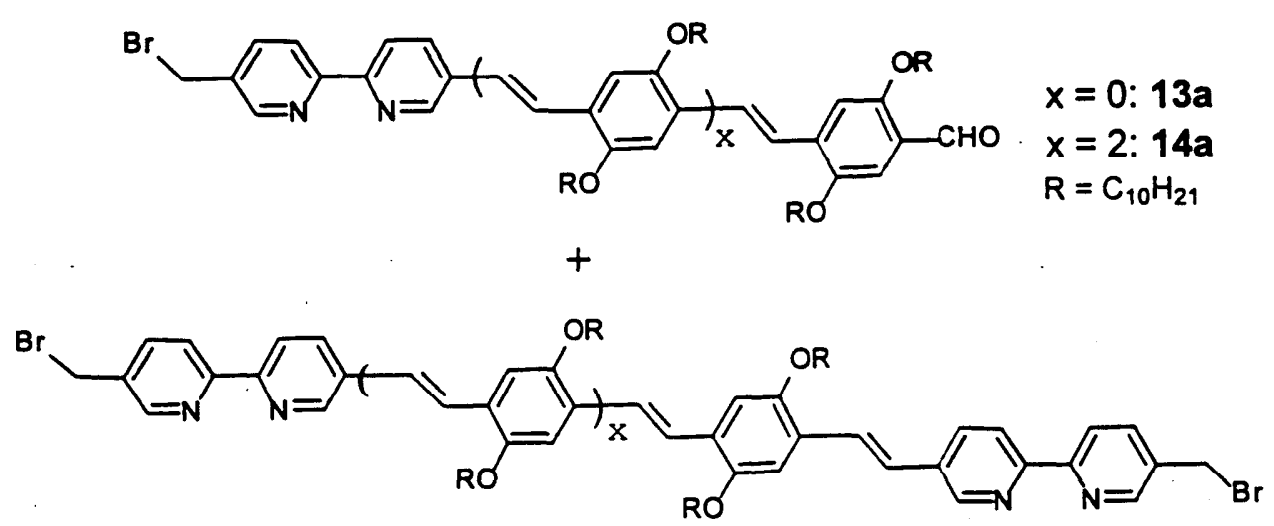

$$
\begin{aligned}
& x=0: 13 b \\
& x=2: 14 b \\
& R=C_{10} H_{21}
\end{aligned}
$$

Figure 4. Synthetic scheme for polymer precursors.

provided a key intermediate 5 ( $86 \%$ yield). The two bromomethylenes were confirmed to be at 2- and 5-positions, as opposed to 2- and 3-, or 2- and 6-positions, by both ${ }^{1} \mathrm{H}-\mathrm{NMR}$ and ${ }^{13} \mathrm{C}-\mathrm{NMR}$ spectra. Attempts at direct conversion of this intermediate to the dialdehyde 8 by known literature procedures were unsuccessful in our hands?' We thus decided to use an acetylation-reduction-oxidation sequence to execute this transformation (i.e. $\mathbf{5} \rightarrow \mathbf{6} \rightarrow \mathbf{7} \rightarrow \mathbf{8}$ ). Although this route took two more steps than a direct conversion, the synthetic efficiency turned out to be extraordinarily good. For instance, the overall yield of 8 starting from 5 in three steps was $\mathbf{8 5 \%}$ The high efficiency of transformation also ensures the ease of isolation and purification at each step. As such, the whole sequence from starting material 3 to dialdehyde 8 can be accomplished with minimal 
chromatographic purification. With intermediate 8 , a triphenylenevinylene dialdehyde 10 was then synthesized. It was accomplished using a double Wittig reaction of 8 with a diphosphonium salt 9 that also was made from compound 5. Like typical Wittig-type reactions, this reaction also yielded a mixture of trans- and cis- vinylene isomers. The isomerization of the mixture to all trans- isomer 10 was achieved by treatment with iodine in dichloromethane at room temperature ( $84 \%$ overall yield).

Synthesis of another building block, namely the phosphonium salts of the 2,2'dipyridine derivatives, is shown in Figure 4. The key precursor 5,5'-bis(bromomethyl)2,2'-bipyridine 11 was prepared from $\beta$-picoline in two steps according to literature procedures. ${ }^{18}$ Further attempts at making its diphosphonium salt in refluxing toluene failed due to the poor solubility of this salt. In fact, it only afforded the monophosphonium salt (12) in the form of a white precipitate in a quantitative yield. At this point, we realized that even though the diphosphonium salt could be made by choosing other solvent systems, it might still be problematic to find an appropriate solvent for a homogenous polymerization reaction between the resultant diphosphonium salt and the dialdehyde under Wittig reaction conditions. On the other hand, if we could take advantage of the formation of mono-phosphonium salt 12 , this problem could be resolved in two ways. For example, from 12 we could synthesize a compound like 13a or 14a that carries a bromomethyl entity at the bipyridine end and a formy1 group at the oligophenylenevinylene end. Further conversion of bromomethyl entity to its phosphonium salt then would enable this bifunctionalized compound to self-polymerize under Wittig reaction conditions. Also, the mono-phosphonium salt 12 can be transformed to dibromomethyl-bipyridine capped phenylenevinylene oligomers. such as compounds $\mathbf{1 3 b}$ and 14b. Their diphosphonium salt counterparts should be quite soluble owing to the presence of solublizing groups on the phenylenevinylene moieties. In light of these considerations, we then chose to react 12 with equivalent dialdehydes 8 and $\mathbf{1 0}$, respectively. As such, in the case of using aldehyde 8 compounds, 13a and 13b were obtained in a one-pot reaction in the yields of $49 \%$ and $11 \%$. respectively. Likewise, compounds 14a and 14b were obtained when aldehyde 10 was used (25\% and $35 \%$ yields, respectively). Again, these Wittig reaction products were mixtures of trans- and 


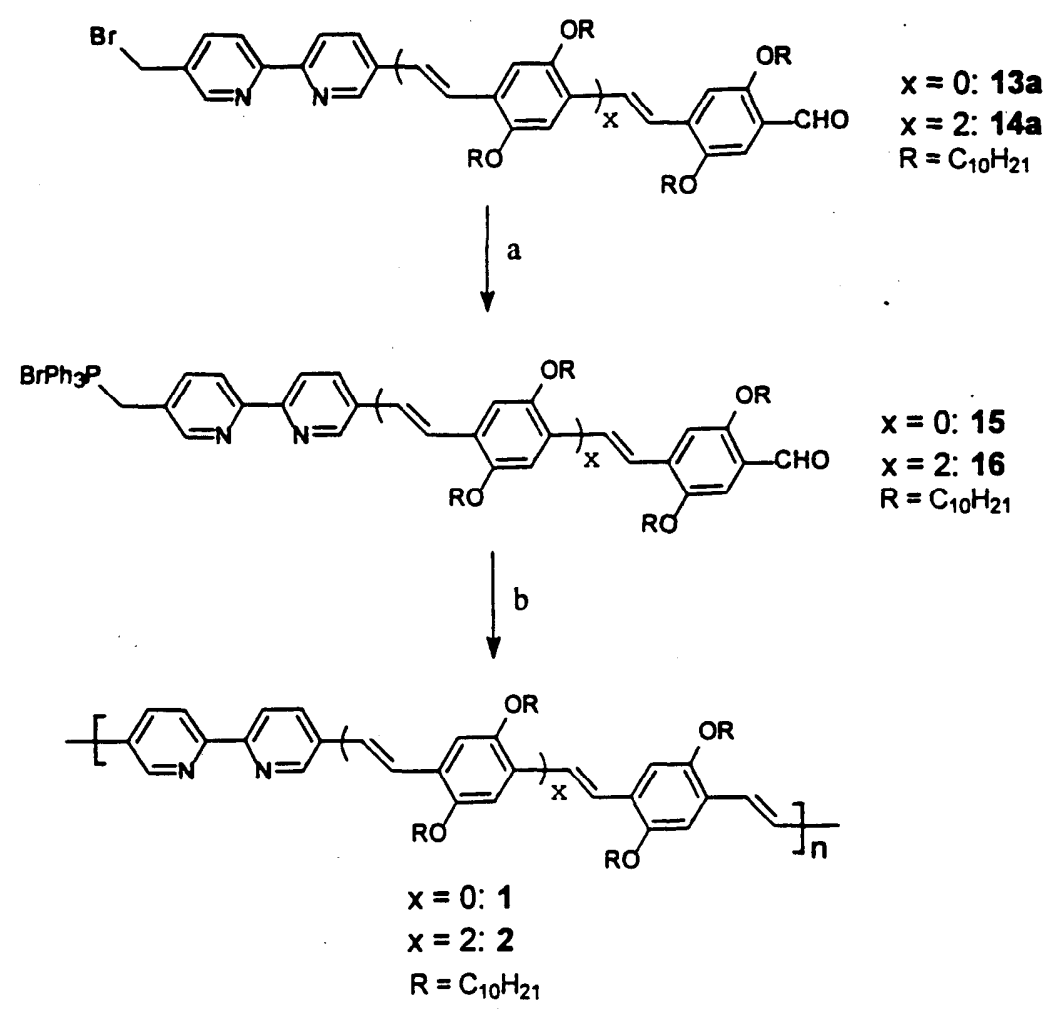

Figure 5. Synthetic scheme for polymers 1 and 2.

cis- isomers. The cis- to trans- isomerizations were achieved by heating the mixture in toluene at reflux in the presence of p-toluenesulfonic acid.

Syntheses of polymers 1 and 2 are highlighted in Figure 5. The stepwise nature of this polymerization reaction dictates that the molecular weight of the polymer depends on the reaction time and the strict 1:1 ratio of two reactants. This latter requirement is normally hard to meet in practice, especially when the polymerization is carried out on a micro-scale. In this regard, it seems that using a building block containing both phosphonium salt and an aldehyde functional group, such as compounds 13a and 14a, for the polymerization may be more advantageous over using two building blocks, like compounds $\mathbf{1 3 b}$ and $\mathbf{1 4 b}$. We thus selected this strategy as an alternative synthetic route for polymers $\mathbf{1}$ and 2. The phosphonium salts 15 and $\mathbf{1 6}$ were made from their precursors 13a and 14a, respectively. Subsequent polymerization was then initiated upon addition of base (LiOEt). In this way, polymer 1 was obtained in $89 \%$ yield $\left(M_{n}=6400\right)$ and polymer 2 made in $92 \%$ yield $\left(M_{n}=22000\right)$. 


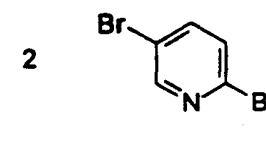

17

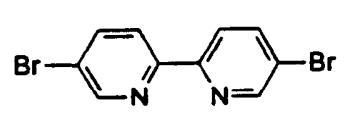

18

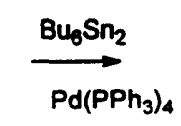

$\underset{\mathrm{Pd}\left(\mathrm{PPh}_{3}\right)_{4}}{\overbrace{\mathrm{SnBu}_{3}}}$

$\left.\mathrm{Pd}_{(\mathrm{PP}}\right)_{4}$<smiles>Brc1ccc(-c2ccc(Br)cn2)nc1</smiles>

18

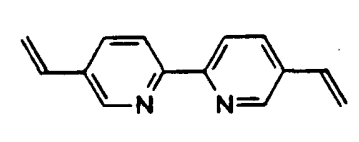

19

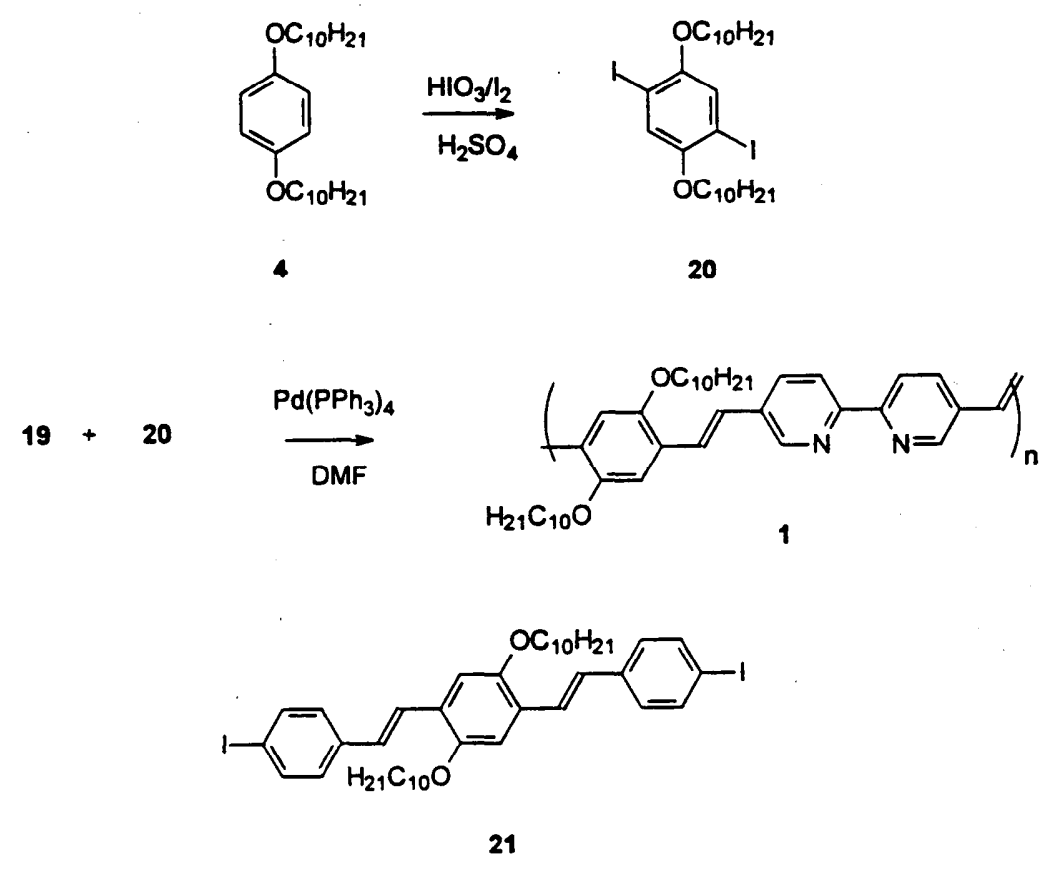

Figure 6. Synthetic scheme for polymers 1 and 2 based on $\operatorname{Pd}(0)$ coupling chemistry.

A more direct route to these polymers was developed that uses far fewer steps with higher yield reactions to produce polymers with higher molecular weights and better film producing properties necessary for sensor applications. The chemistry is illustrated in Figure 6. We have developed a unique one-step preparation of the key intermediate 5,5'-dibromo-2,2'-bipyridyl, 18 by coupling 2 molecules of commercially available 2,5dibromopyridine, 17 at their 2-positions using a $\operatorname{Pd}(0)$ catalyst. In this case the dibromo compound 18 is treated with $\mathrm{Bu}_{6} \mathrm{Sn}_{2}$ and a catalytic amount of $\mathrm{Pd}\left(\mathrm{PPh}_{3}\right)_{4}$ in refluxing 
toluene to give about a $65 \%$ yield of the desired product. The 5,5'-dibromo-2,2'bipyridyl was further functionalized with an excess of vinyltributyltin and $\mathrm{Pd}\left(\mathrm{PPh}_{3}\right)_{4}$ catalyst to yield the corresponding 5,5'-divinyl derivative, 19 in $80 \%$ yield. The dialkoxybenzene components of the polymers were synthesized starting from 1,4didecyloxybenzene, 4 and subjecting it to diiodination using $\mathrm{HIO}_{3} / \mathrm{I}_{2} / \mathrm{H}_{2}$ to yield the 2,5diiodo- 1,4-didecyloxybenzene, 20. Polymer 1 can be synthesized in approximately $80 \%$ overall yield by refluxing equimolar amounts of 19 and 20 in DMF in the presence of a catalytic amount of $\mathrm{Pd}\left(\mathrm{PPh}_{3}\right)_{4}$. The molecular weight of this polymer is 35,000 .

To improve the synthesis of polymer 2 bisphosphonium salt 9 was reacted with 2 moles of p-iodobenzaldehyde to give a 65\% yield of phenylenevinylene oligomer 21 . The diiodo oligomer, 21 was reacted with an equimolar amount of 19 in DMF in the presence of a catalytic amount of $\mathrm{Pd}\left(\mathrm{PPh}_{3}\right)_{4}$ to yield polymer 2. The molecular weight of this polymer is 40,000 .

A synthesis of a new type of polymer that promises to give us the versatility in design modification that we need for rapid attachment of ligands that bind heavy metals, such as lead and plutonium, was developed. The synthetic scheme for this polymer is given in Figure 7. Hydroquinone, 3 was monoalkylated with 1-bromo-2-ethylhexane to give the monoether, 22 in $85 \%$ yield. To make comparisons with polymers 1 and 2 a 2,2'-bipyridyl ligand was chosen for the initial synthesis. In this case 4,4'-dimethyl-2,2'bipyridyl, 23 can be monobrominated with $\mathrm{N}$-bromosuccinimide in $\mathrm{CCl}_{4}$ to give a $50 \%$ yield of 4-bromomethyl-4'-methyl-2,2'-bipyridyl, 24. Bromo derivative 24 can be used to alkylate the remaining hydroxy group on monoether 22 , using $\mathrm{K}_{2} \mathrm{CO}_{3}$ in refluxing DMF to yield the bipyridyl derivative 25 . This derivative is bisbromomethylated using $1 \mathrm{HBr}$ in acetic acid and paraformaldehyde to yield derivative 26. Based catalyzed polymerization of 26 using potassium t-butoxide in THF furnishes the desired polymer, 27. In this polymer binding of metals requires two adjacent bipyridyl groups to cooperate in the binding. This results in a twisting distortion of the $\pi$ conjugated pathway in the polymeric backbone and results in a blue shift of the optical absorption and emission from polymer 27 . This effect complements that observed for polymer 1 in which a red shift is observed upon metal binding. 

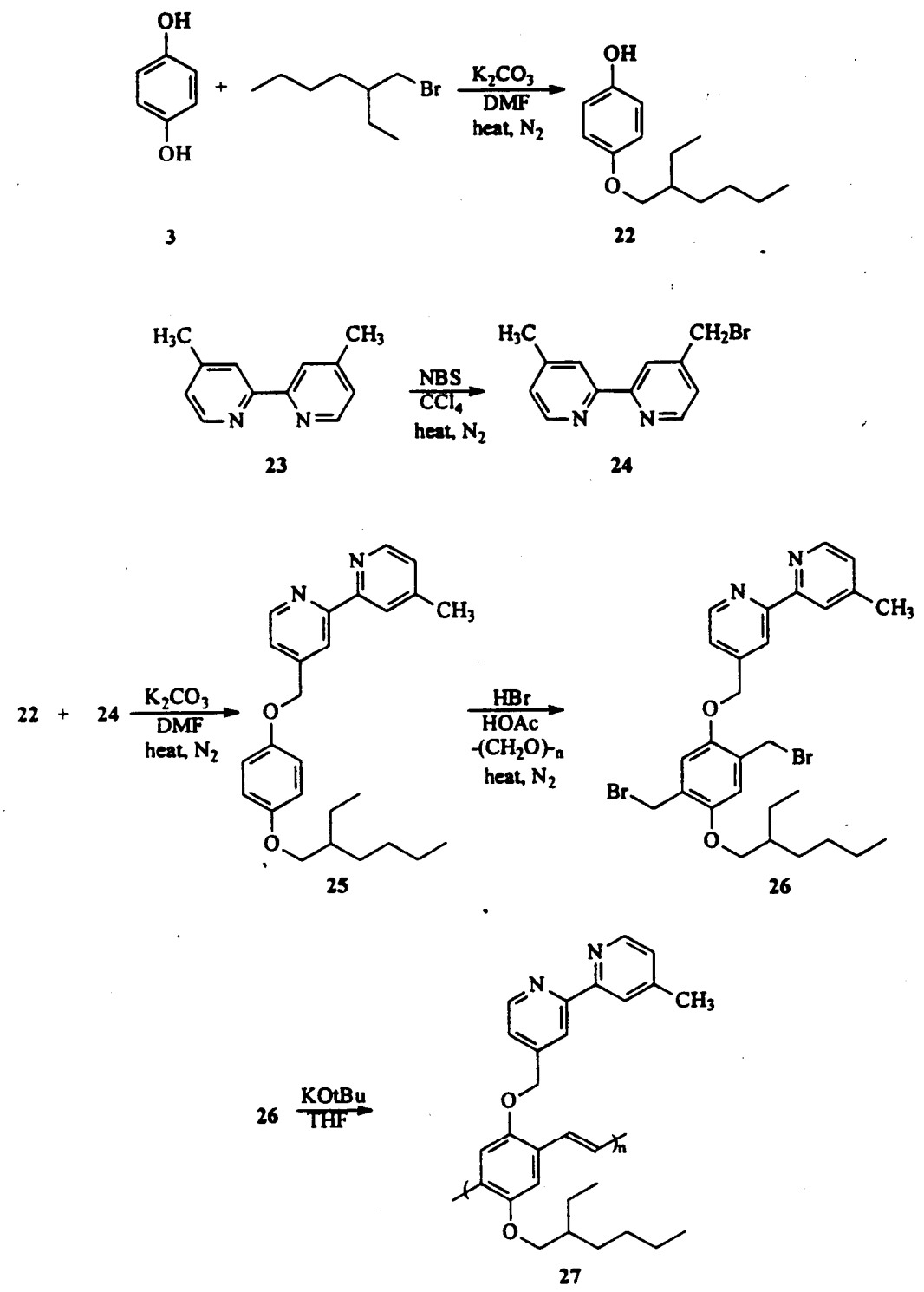

Figure 7. Synthetic scheme for side chain ligand substituted poly(phenylenevinylene) polymers.

Monoether 20 is a versatile reagent that can be used to append other ligands to a conjugated polymer backbone. The Raymond group at UC Berkeley, who are our collaborative partners in this project, are preparing ligands that are specific for iron, plutonium (IV), uranyl, and lead ions, which are highly suitable for incorporation as molecular recognition sites in the luminscent, conductive polymers. This work has centered on making chemical modifications to the functional groups on the important hydroxypyridonate (HOPO) ligand that they have developed for chelation of iron and plutonium (IV) to be compatible with the luminescent polymers. In addition, this group 
has investigated ways in which to functionalize the sequestering agents that they have developed for uranyl ions ${ }^{18}$ with the goal of providing a ligand that will make the properties of the conjugated polymers sensitive to the presence of the uranyl ion. Finally, the Raymond group is investigating new ligands that make use of the stereochemical coordination properties of lead ions to produce polymer-compatible ligands that are sensitive to lead.

The ideal chemical sensor measures the in situ concentration of analyte continuously, reversibly, inexpensively, and rapidly. In most situations where measurements are taken, it is important to measure several parameters simultaneously. One of the benefits accrued by using an imaging fiber in the fabrication of a multianalyte fiber-optic chemical sensor is the ability to selectively address the separate optical pathways of the imaging fiber during the indicator immobilization procedure. Work in the Walt laboratory at Tufts has focused on developing photoinitiated polymerization reactions that can accommodate the presence of the poly(phenylenevinylene) polymers at well separated spots on the tip of a single optical imaging fiber.

A second approach that has been investigated involves photoimmobilization of an indicator containing a photoactivated functional group. In this case, selective photo excitation of a particular region of the fiber activates the indicator which then binds to a polymer layer disposed uniformly over the entire fiber surface. Many distinct sensing regions can be photodeposited onto a single 350- $\mu$ m-diameter imaging fiber with the size of the pinhole and the objective's magnification controlling the size of each sensing region. Using a charge coupled device, the fluorescence from each of the different sensing regions immobilized on the imaging fiber can be spatially resolved. ${ }^{17}$ The creation of spatially discrete sensing sites on a single optical sensor solves many of the problems associated with designing multianalyte optical sensors such as spectral overlap of multiple indicators and the need to use individual optical fibers for each analyte. In addition, when these discrete sensing regions are immobilized around the periphery of the imaging fiber's distal face, the bare central region can be used either to view the sample or as a background measurement. Similar tactics are being used to immobilize the 
conjugated polymers possessing molecular recognition sites onto the end of the fiber optic bundle.

\section{References}

1)Czarnik, A. W. Fluorescent chemosensors for ion and molecular recognition; 538 ed.;

Czamik, A. W., Ed.; American Chemical Society: Washington, DC, 1993.

2)Swager, T. M.; Marsella, M. J. J. Adv. Mater. 1994, 6,595.

3)Fabbrizzi, L.; Poggi, A. Chem. Soc. Res. 1995, 191.

4)Josowicz, M. Analyst 1995, 120, 1019.

5)Marquis, D.; Desvergne, J.-P.; Bouas-Laurent, H. J. Org. Chem. 1995, 60,7984.

6)Bobacka, J.; Lewenstam, A.; Ivaska, A. Tanata 1993, 40, 1437.

7)Marsella, M. J.; Swager, T. M. J. Am. Chem. Soc. 1993, 115, 12214.

8)Marsella, M. J.; Newland, P. J.; Carrol, P. J.; Swager, t. M. J. Am. Chem. Soc. 1995, $117,9842$.

9)Bauerle, P.; Scheib, S. Adv. Mater. 1993, 5, 848.

10)McCullough, R. D.; Williams, S. P. Chem. Mater. 1995, 7, 2001.

11)Sable, E.; Handel, E.; L'Her, M. Electrochim. Acta 1991, 36, 15.

12)Brockmann, T. W.; Tour, J. M. J: Am. Chem. Soc. 1995, 117, 4437.

13)Raymond, K. N.; Telford, J. R.; Raymond, K. N.; Telford, J. R., Ed.; Kluwer Academic: The Netherlands, 1995; Vol. 459, pp 25-37.

14)Raymond, K. N.; Xu, J. ; Raymond, K. N.; Xu, J., Ed.; CRC Press, Inc.: Boca Baton, Florida, 1994, pp 307-327.

15)Raymond, K. N.; Durbin, P. W. ; Raymond, K. N.; Durbin, P. W., Ed.: Richland, Washington, 1993; Vol. Publication PNL-SA-21775, pp LBL Number 31872.

16)Bamard, S. M.; Walt, D. R. Nature 1991, 353, 338-340.

17)Bronk, K. S.; Walt, D. R. Anal. Chem. 1994, 66, 3519-3520.

18)Walton, P. H.; Raymond, K. N. Inorg. Chim. Acta 1996, 240, 593-601. 